Artículo original

\title{
Análisis multiespectral y digital de los nevos melanocíticos adquiridos
}

\author{
Ariana C. Di Lorenzo V. ${ }^{1 * *}$, Reimer A. Romero H. ${ }^{1}$, Aarón Muñoz T. ${ }^{1}$, Sandra C. Vivas ${ }^{1,2}$, Irene Paredes ${ }^{1,2}$ \\ ${ }^{1}$ Departamento de Física y Centro de Investigaciones Médicas y \\ Biotecnológicas de la Universidad de Carabobo (CIMBUC), Valencia, Carabobo, Venezuela \\ ${ }^{2}$ Postgrado de Dermatología, Centro Hospitalario Enrique Tejera, Centro de Investigaciones Médicas \\ y Biotecnológicas de la Universidad de Carabobo (CIMBUC), Valencia, Carabobo, Venezuela
}

\begin{abstract}
Resumen
El objetivo del presente trabajo fue encontrar las diferencias entre los distintos tipos de nevos melanocíticos adquiridos con base en la información extraída de las imágenes multiespectrales. La dimensión fractal y la entropía se escogieron como medidas para distinguir entre los nevos (146 lesiones). El análisis de varianza (ANOVA unifactorial) considerando la dimensión fractal mostró diferencias significativas $(p<0,05)$ entre los grupos, con mayor significación en la longitud de onda azul ( $p=7,87 \mathrm{e}-08)$, y menor en la roja. La medida de la entropía se comparada entre los pares de grupos mediante la prueba $\mathrm{T}$ de Student, y se encontraron diferencias entre los lunares benignos (U,C,I) y los displásicos (D), específicamente en los canales cyan $\left(\mathrm{p}_{\mathrm{UD}}=0,002, \mathrm{p}_{\mathrm{CD}}=0,023, \mathrm{p}_{\mathrm{ID}}=0,012\right)$ y rojos $\left(\mathrm{p}_{\mathrm{UD}}=0,0001, \mathrm{p}_{\mathrm{CD}}=2,55 \mathrm{E}-5, \mathrm{p}_{\mathrm{ID}}=0,005\right)$. El uso de imágenes multiespectrales, conjuntamente con las medidas de ordenamiento (dimensión fractal y entropía), permite caracterizar y diferenciar los grupos de nevos melanocíticos adquiridos, lo cual contribuiría al desarrollo de un tipo de evaluación dermatológica no invasiva y concluyente del ordenamiento de los nidos de melanina.
\end{abstract}

Palabras clave: Dimensión fractal; Multiespectral; Entropía; Nevos; Dermatoscopía.

Multiespectral and digital analysis of acquired melanocytic nevi

\begin{abstract}
The main objective of the present work was to find differences between different types of acquired melanocytic nevi on 146 lesions. Fractal dimension and entropy were obtained from multispectral images. We found significant differences in fractal dimension measures using an analysis of variance (one-way ANOVA) $(\mathrm{p}<0.05)$, with $\mathrm{p}=7.87 \mathrm{e}-08$ at the blue wavelength. The entropy mean of all NMA pairs was compared using a T-Student test; we registered a high significance in the cyan channels $\left(\mathrm{p}_{\mathrm{UD}}=0.002, \mathrm{p}_{\mathrm{CD}}=0.023, \mathrm{p}_{\mathrm{ID}}=0.012\right)$ and in the red ones $\left(\mathrm{p}_{\mathrm{UD}}=0.0001\right.$, $\mathrm{p}_{\mathrm{CD}}=2,55 \mathrm{E}-5, \mathrm{p}_{\mathrm{ID}}=0.005$ ). Multispectral images together with order measures (fractal dimension and entropy) is a tool to characterize and distinguish NMA groups and a way towards developing a non-invasive dermatologic test based on melanin distribution in NMAs.
\end{abstract}

Key words: Fractal dimension; Multiespectral; Entropy; Nevi; Dermastocopy.

\section{Introducción}

En dermatología el color de la piel y el de las lesiones cutáneas contienen información vital para el diagnóstico. La evaluación del estado de la piel en tiempo real todavía es una utopía de la medicina de especial importancia para la solución de los problemas de salud de la piel en el menor tiempo posible.

El análisis de la interacción de la luz con la piel humana es una alternativa no invasiva para el diagnóstico de tejidos anómalos y lesiones cutáneas, tales como el melanoma en sus diferentes estados (Paredes, et al., 2016).

Aunque el melanoma cutáneo afecta principalmente a los caucásicos, su incidencia varía según las regiones geográficas. Son pocos los datos clínicos e histopatológicos disponibles de pacientes con melanoma en Latinoamérica.
Los datos reales de incidencia del melanoma son escasos $\mathrm{y}$, en general, provienen de estudios pequeños realizados en una sola institución (Schmerling, et al., 2011).

La incidencia anual estimada de melanoma en Latinoamérica no supera los cinco casos anuales por cada 100.000 habitantes, siendo Uruguay el país que ocupa el primer lugar, con una incidencia de 4,1, seguido de Colombia, Argentina y Brasil, con una incidencia de 3,3, 2,9 y 2,8, respectivamente. En Venezuela la incidencia es de 1,1 y es uno de los países con menor incidencia en Latinoamérica. Estas incidencias siguen siendo bajas comparadas con las de otros

\footnotetext{
*Correspondencia:

Ariana Caridad Di Lorenzo Valdes, Aridilorenzo@gmail.com

Recibido: 22 de enero de 2018

Aceptado: 27 de abril de 2018

Editor: Juana Ángel
} 
países como Australia y Nueva Zelanda, con 34,9 y 35,8, respectivamente, según los datos del Centro Internacional de Investigaciones sobre el Cáncer (2008) y del Atlas del cáncer (2008) (Schmerling, 2011).

El melanoma aparece inicialmente como una mancha pigmentada muy parecida a los nevos melanocíticos adquiridos, comúnmente conocidos como lunares.

Los nevos melanocíticos adquiridos (NMA) son la neoplasia más común en el ser humano; existen reportes en los que su prevalencia en la población oscila entre el 90 y el $95 \%$ y varía según la edad, la etnia, y los factores ambientales y genéticos. El número total de lunares aumenta con la edad pero presenta un crecimiento rápido en la pubertad, con un pico máximo en la tercera década de la vida en ambos sexos. Asimismo, se ha observado un mayor número de lunares en familiares de pacientes con antecedentes de cáncer de piel y en quienes se han expuesto excesivamente a radiaciones ultravioleta, por lo que es lógico pensar que cada vez son más frecuentes las consultas relacionadas con su potencial de malignidad y con la necesidad de tratamiento (Wolff, et al., 2010; Moreno \& Saucedo, 2012; Sortino-Rachou, et al., 2011).

Los NMA son pequeñas manchas pigmentadas que se originan por la proliferación anormal pero benigna de células llamadas melanocitos, los cuales tienden a agruparse en nidos de melanocitos y afectan las diferentes estructuras de la piel (Rex \& Ferrándiz, 2007).

Los NMA se clasifican en nevos de unión, nevos compuestos, nevos intradérmicos y nevos displásicos, y cada tipo tiene características visuales e histológicas diferentes. Un nevus displásico puede convertirse en una lesión maligna y cuantos más tenga una persona, mayor es el riesgo de desarrollarla. El criterio clínico de diagnóstico de estas lesiones es netamente visual y sigue la conocida regla basada en la observación de la asimetría, el borde, el color y el diámetro de la lesión (ABCD) (Ruela, et al., 2015).

Sin embargo, frente a una lesión sospechosa de ser maligna debe hacerse un estudio histológico, el cual es costoso e invasivo, por lo que varios autores han considerado la digitalización de la regla $\mathrm{ABCD}$ (Isasi, et al., 2011; Chang, et al., 2013), la cual consiste en segmentar la lesión, establecer sus características y clasificar las imágenes en rojo, verde y azul (RGB) que se obtienen con dispositivos de carga acopalada (Charge Coupled Device, CCD) o con el semiconductor complementario de óxido metálico (complementary metal-oxide-semiconductor, CMOS) (Kapsokalyvas, et al., 2013; Kim, et al., 2016).

Muchos de estos trabajos se han desarrollado como respuesta a la alta incidencia de melanomas y gracias al uso de máquinas de aprendizaje para distinguir los melanomas de otro tipo de lesiones benignas (Amelard, et al., 2014)

Algunos autores recurren a análisis estadísticos como el ANOVA, la prueba T de Student, y el test de diferencia significativa mínima (least significant difference, LSD) de Fisher para determinar las diferencias entre los tipos de lesión. En este sentido, se han utilizado los espectros infrarrojos (McIntosh, et al., 2001) para determinar diferencias significativas entre los distintos tipos de lesiones, entre ellas, los nevos displásicos. Asimismo, mediante la observación de las imágenes de los nevos melanocíticos pueden establecerse características como la dimensión fractal para diferenciarlos de los menalonas (Manousakia, et al., 2016).

La interacción de la luz con la piel humana representa una alternativa no invasiva para el diagnóstico de tejidos anómalos y lesiones cutáneas en sus diferentes estados. Una de estas técnicas es el estudio de las imágenes multiespectrales (Vivas \& Muñoz, 2016; Kim, et al., 2016).

Las imágenes multiespectrales, que extienden el número de canales de imagen más allá de los tres convencionales (rojo, verde y azul), han demostrado ser útiles para una amplia gama de aplicaciones y, a diferencia de las imágenes comunes, revelan más de lo que permite la visión humana. El sistema de imágenes multiespectrales se basa en la utilización de diferentes longitudes de ondas del espectro visible para aumentar el contraste de los diferentes cromóforos que componen las capas de la piel (Air University, 2003). De esta forma, la luz penetra en la piel a diferentes profundidades, lo que permite obtener imágenes diferentes a las comunes. Cada segmento de imagen representa una longitud de onda que informa sobre el contenido de los elementos de absorción de la piel, por lo pueden ser específicos para cada segmento del tejido.

La secuencia de imágenes se puede superponer para obtener una imagen bidimensional de la lesión (Vivas \& Muñoz, 2016). Asimismo, algunas estructuras pueden discernirse según la absorción y el esparcimiento de los cromóforos presentes, lo cual facilitaría el diagnóstico a partir de la morfología patológica (Kapsokalyvas, et al., 2013).

El estudio de las imágenes multiespectrales se basa en diferentes fenómenos físicos como la absorción, el esparcimiento, la reflexión y la transmisión, los cuales ocurren cuando una onda electromagnética incide sobre las diferentes capas de la piel (epidermis, dermis, hipodermis) con una longitud medible (de 400 a $700 \mathrm{~nm}$ ) para luego emerger del interior de la piel como luz reflejada de forma difusa (Vivas \& Muñoz, 2016).

Con este enfoque, la piel se reconoce como un medio turbio estratificado. Cuando la luz incide sobre los tejidos, la energía que penetra en la piel no se propaga en dirección recta, pues al entrar en contacto con las diferentes estructuras que componen el tegumento, la luz cambia de dirección de propagación, fenómeno este denominado esparcimiento. Los elementos responsables del esparcimiento en la piel son las fibras de colágeno, las organelas celulares, las células, los folículos pilosos y las glándulas sudoríparas (Fodor, et al., 2012).

Para obtener las imágenes multiespectrales nos apoyamos en la dermatoscopia, técnica diagnóstica no invasiva que se basa en la observación submacroscópica de lesiones cutáneas melanocíticas y no melanocíticas. Mediante la 
utilización de un sistema óptico, llamado dermatoscopio, se amplifica la imagen por medio de un lente de aumento y se ilumina la piel con un sistema de iluminación multiespectral, consiguiendo así la observación de estructuras epidérmicas y dérmicas invisibles a simple vista. Este procedimiento se debe considerar como un paso intermedio entre la observación clínica y el estudio histopatológico de una lesión (De Giorgi, et al., 2004; Argenziano, et al., 2001).

El presente trabajo tiene como objetivo encontrar diferencias entre los distintos tipos de nevos melanocíticos adquiridos con base en la información proveniente de las imágenes multiespectrales. Dado que estas imágenes cuantifican la energía absorbida por los cromóforos presentes en la piel, lo que depende de la longitud de onda con la que se iluminen, se buscan pistas sobre el ordenamiento de los cromóforos asociados al tipo de nevo. En este sentido, se considera que el orden de los niveles de grises y su distribución en la imagen son cuantificables a través de dos medidas: la dimensión fractal y la entropía. Ello permite reconocer no solo la malignidad (nevo displásico) de la lesión, sino también su avance con respecto al resto de los lunares al verificar si son de tipo unión, compuesto o intradérmico.

\section{Detección de las características a partir de las imágenes digitales}

Una imagen puede definirse como una función bidimensional de la intensidad de la luz $f(x, y)$, donde $x$ y $y$ representan las coordenadas espaciales y el valor de $f$ en un punto cualquiera $(x, y)$ proporcional al brillo o nivel de gris de la imagen en él. Una imagen digital es una imagen $f(x, y)$ que se ha discretizado tanto en las coordenadas espaciales y en el brillo, y puede considerarse como una matriz cuyos índices de fila y de columna identifican un punto de la imagen, en tanto que el valor del correspondiente elemento de la matriz indica el nivel de gris en ese punto.

Una imagen constituye una colección de características visuales que describen su contenido. La detección de información cuantitativa permite diferenciar una clase de objetos de otra (Ruiz, et al., 2010).

Con el fin de caracterizar los diferentes tipos de nevos melanocíticos adquiridos se establecieron sus diferentes características mediante el análisis fractal y el análisis de la entropía.

\section{Análisis fractal}

El concepto fractal se ha utilizado en muchos estudios de imágenes médicas, como la morfogénesis pulmonar, las mamografías y las imágenes ultrasónicas de hígado (Falconer, 2007), así como en imágenes dermatoscópicas (Cavalcanti \& Scharcanski, 2014; Manousakia, et al., 2016).

Los NMA son elementos de la naturaleza que pueden describirse mediante la geometría fractal. El análisis fractal es el conjunto de procedimientos matemáticos utilizados para determinar la dimensión fractal de un objeto con el menor error posible y esta es la medida numérica adimensional del grado de irregularidad de un objeto.
El método más usado de determinación de la dimensión fractal es la llamada dimensión de Minkowski-Bouligand, también conocida como dimensión de Minkowski o dimensión de conteo de cajas. Al superponer el fractal en una cuadrícula de espacio uniforme, se cuenta la cantidad de cuadros necesarios para cubrir el objeto. La dimensión de conteo de cajas se calcula al ver cómo cambia este número a medida que hacemos la cuadrícula más fina. Asumiendo $N_{S}$ como el número de cajas del lado $S$ requeridas para cubrir el objeto, la dimensión fractal $\mathrm{D}$ se define como:

$$
D=\lim _{S \rightarrow 0}\left(\frac{\operatorname{Ln}\left(N_{S}\right)}{\operatorname{Ln}(1 / S)}\right)
$$

donde D es la dimensión fractal, $N_{s}$ es el número de piezas para cubrir el objeto y $S$ es la escala del objeto. En la Figura $2 \mathrm{f}$ puede verse la última fase del análisis fractal.

El aumento de la irregularidad del objeto incide en el de la dimensión fractal. Esto corresponde a nuestra noción intuitiva de rugosidad o textura: cuanto más bajo sea el valor de $\mathrm{D}$, más suave será el objeto, y cuanto mayor sea el valor de D, más áspero (Falconer, 2007).

Manausaki, et al. (2016) consideraron la medida de la dimensión fractal para distinguir 23 melanomas y 109 nevos melanocíticos adquiridos (44 displásicos y 65 nevos melanocíticos comunes) a partir de imágenes RGB. Sus resultados no mostraron una diferencia significativa $(p=0,359)$ entre los nevos displásicos y los nevos melanocíticos comunes.

\section{Análisis de la entropía}

Al comparar imágenes es importante tener algún tipo de medida de la cantidad de información disponible en ellas. Shannon presentó una forma de medir la cantidad de información que contiene una variable aleatoria, conocida como entropía de Shannon, según se define en la ecuación 2.

$$
E=\sum_{i=1}^{i=N} P_{i} \log \left(1 / P_{i}\right)=-\sum_{i=1}^{i=N} P_{i} \log \left(P_{i}\right)
$$

donde $P(i)$ es la probabilidad de que ocurra un evento de la $i$-clase, $N$ es el número total de clases, e $i$ es la $i$-clase. En el caso de las imágenes, $P(i)$ es la probabilidad de que se obtenga un nivel de gris específico $i$, y $N$ el número total de grises en la imagen, que en el caso de una imagen de 8 bits es de 256 niveles de grises. El término $\log (1 / P(i))$ implica que la cantidad de información contenida en una imagen con distribución de grises $P(i)$ está inversamente relacionada con la probabilidad de aparición del nivel de gris $i$. Debe señalarse que la entropía de Shannon también puede concebirse como una medida de incertidumbre, la cual depende no solo del número de posibles niveles de gris sino también de la probabilidad de que estos ocurran. La entropía es máxima cuando todos los niveles tienen la misma probabilidad de ocurrir (Brieva \& Montes, 1995; Pluim, et al., 2003). 
Así, una imagen con una gran cantidad de pixeles con la misma intensidad tiene poca entropía, en tanto que con una distribución uniforme de los valores de gris se obtiene una entropía elevada. En conclusión, la entropía de Shannon también constituye una medida de la distribución de la probabilidad de los valores de gris (Pluim, et al., 2003).

\section{Metodología}

Este proyecto de investigación se llevó a cabo en el Centro de Investigaciones Médicas y Biotecnológicas de la Universidad de Carabobo, Venezuela. La población incluyó sujetos a quienes médicos especialistas en dermatología diagnosticaron en un examen visual utilizando el método de ABCD; el diagnóstico definitivo se hizo mediante histopatología. Se pudo comparar la eficiencia de los métodos visuales con el diagnóstico histopatológico para clasificar el tipo de lunar, garantizando la evaluación independiente y cegada de la prueba que se pretendía validar y la técnica diagnóstica con la que se comparó. Se incluyeron en el estudio 146 muestras de lesiones melanocíticas adquiridas provenientes de 83 pacientes, todas diagnosticadas mediante histopatología y clasificadas posteriormente según los cuatro tipos de nevos melanocíticos adquiridos.

\section{Obtención de las imágenes multiespectrales}

Las imágenes multiespectrales se obtuvieron utilizando un dermatoscopio multiespectral construido en el Centro de Investigaciones Médicas y Biotecnológicas de la Universidad de Carabobo (CIMBUC) (Muñoz, 2014). Dicho equipo está constituido por ocho diodos emisores de luz (light-emitting diode, LED) tricromáticos de $3 \mathrm{~mm}$ : azul $(470 \mathrm{~nm})$, verde $(580 \mathrm{~nm})$ y rojo $(660 \mathrm{~nm})$, con una lente de $25 \mathrm{~mm}$ y un aumento variable de $1 \mathrm{X}$ a $4 \mathrm{X}$. Posee también una rosca que permite cambiar la longitud de onda emitida. Para obtener las imágenes, el dermatoscopio se conecta a un computador y mediante el programa de acceso libre ImageJ
(Image Processing and Analysis in Java) permite visualizar y guardar las imágenes observadas. El especialista coloca el dermatoscopio sobre la lesión a estudiar y selecciona una de las longitudes de onda para visualizar la imagen de la lesión incidida por dicha longitud de onda a través del ImageJ. El proceso se puede hacer con las seis longitudes de onda que permite el dermatoscopio con la ayuda de una cámara CCD de 3.0 megapixeles. Las diferentes bandas o longitudes de onda de la luz penetran en la piel a distintas profundidades, y así se obtienen imágenes secuenciales de la misma lesión para el mapeo distante e in vivo de cromóforos de la piel mediante el análisis de los datos espectrales reflejados en cada pixel de la imagen (Figura 1).

\section{Procesamiento de imágenes multiespectrales}

Antes del procesamiento se obtiene la imagen en escala de grises y la imagen binaria. La primera debe resaltar las variaciones de brillo en un mismo tipo de nevo melanocítico adquirido como efecto de la distribución de los nidos de melanina, por lo que se aplicó una ecualización adaptativa del histograma por contraste limitado (Contrast Limited Adaptive Histogram Equalization, CLAHE). La imagen binaria responde a un umbral tal que binariza en 0 el nevo melanocítico adquirido o NMA y en 1 el resto de la piel sana.

El proceso de captura de la imagen del NMA permite obtenerla en RGB para cada longitud de onda. En este caso, se transformó en una imagen en escala de grises mediante el método de promedios ponderados, es decir: $I=r \times R+$ $g \times G+b \times B$, con valores de $(r, g, b)=(0,299,0,587$ y $0,114)$ para aproximarse a la sensibilidad cromática del ojo humano. No se descartó el uso de otros métodos, pero éstos condujeron a resultados poco satisfactorios. El resultado se ecualizó para así evitar la iluminación no homogénea y mejorar el contraste. Por último, se binarizó la imagen empleando un umbral global, lo que implicó asumir histogramas bimodales (Figura $2 \mathrm{~b}$ ).
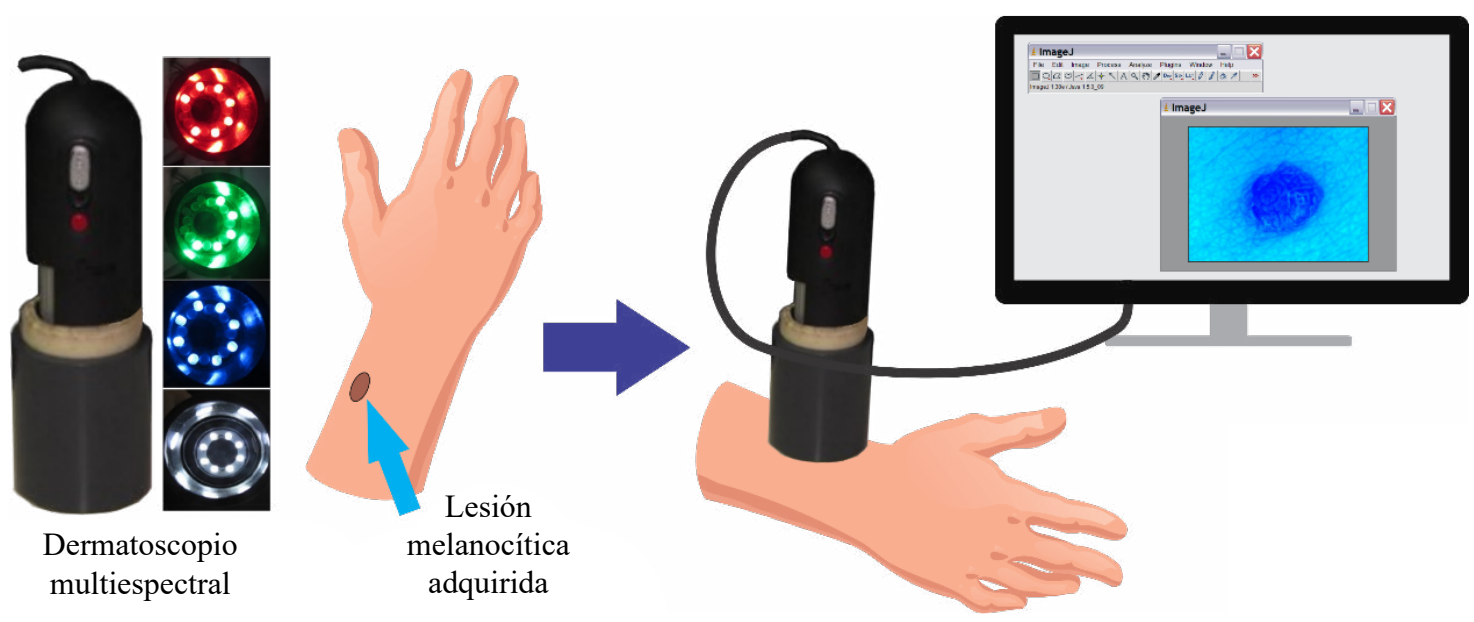

Figura 1. Diagrama del proceso de adquisición de las imágenes multiespectrales utilizando un dermatoscopio multiespectral equipado con LED tricromáticos RGB y una cámara CCD de 3,0 megapixeles conectada a una computadora para capturar las imágenes utilizando el programa Image $\mathrm{J}$ y el paquete Analysis in Java 


\section{Características de las imágenes multiespectrales}

Dimensión fractal. Se diseñó un algoritmo para establecer la dimensión fractal de las imágenes binarias utilizando el método de conteo de cajas en el cálculo de la dimensión fractal (Figura $2 \mathrm{c}$ y d).
Entropía. Se diseñó un algoritmo para calcular la entropía del histograma de las imágenes en escala de grises ecualizadas utilizando el paquete estadístico de python scipy. stats. La imagen proporcionada es un recorte del lunar que incluye solo la lesión. En la Figura 3 se observan cuatro cortes

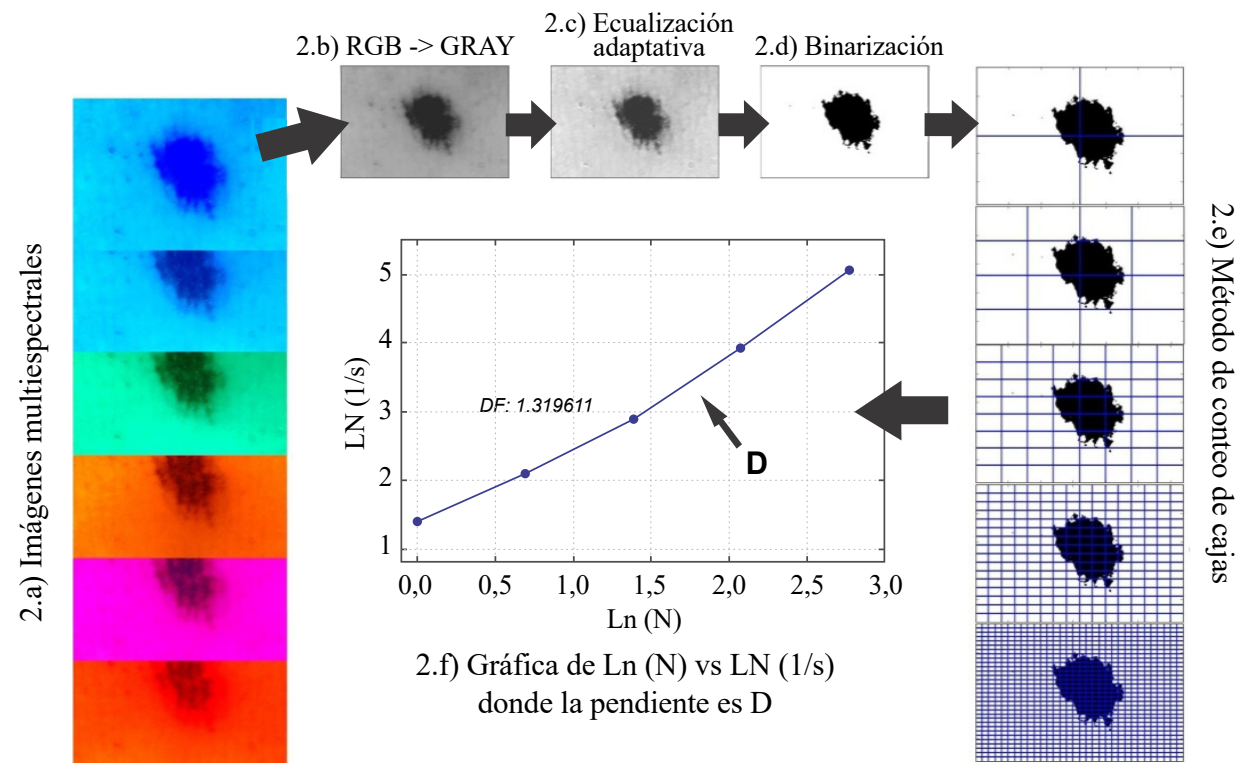

Figura 2. Diagrama del proceso para el cálculo de la dimensión fractal de las imágenes multiespectrales. a) Imágenes multiespectrales. b) Escala de grises. c) Ecualización de la imagen. d) Binarización de la imagen. e) Métodos de conteo de cajas. f) Gráfica de Ln(1/s) Vs. Ln(N), donde el parámetro $\mathrm{D}$ (dimensión fractal) está dado por la pendiente de la recta
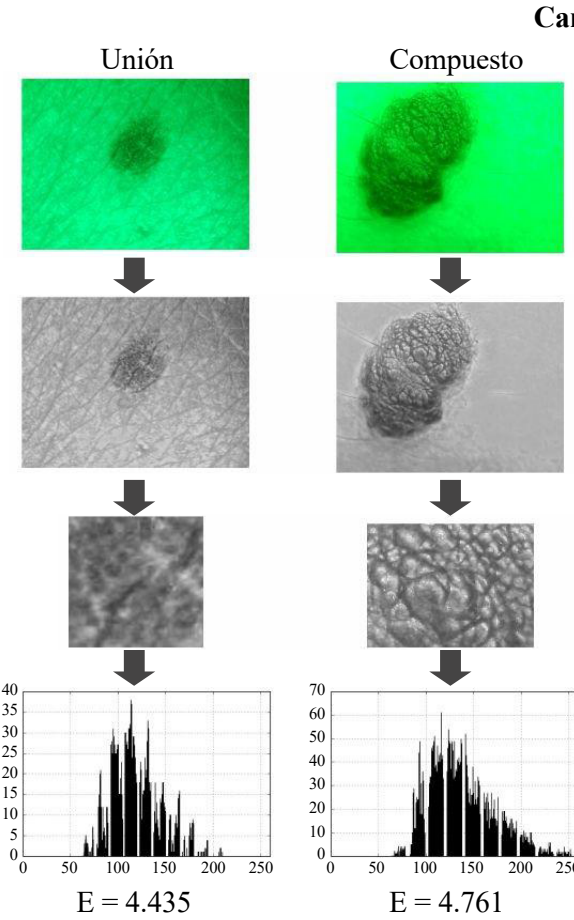

Canal verde
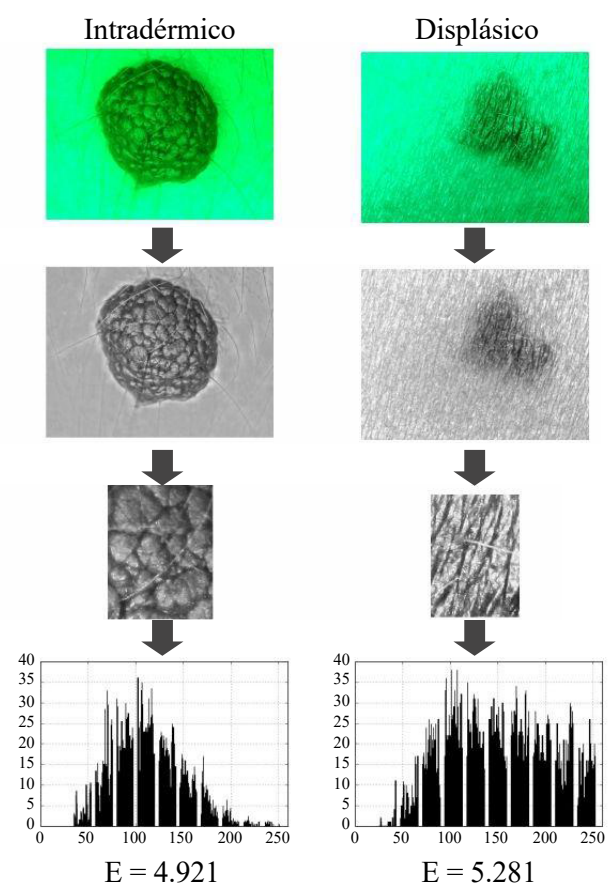

Figura 3. Diagrama de proceso para el cálculo de la entropía de las imágenes multiespectrales. Imágenes multiespectrales, escala de grises, ecualizado, recorte del área de la lesión que se va a estudiar, gráfica del histograma de la imagen de la lesión recortada y por último se calcula la entropía del histograma de la imagen. 

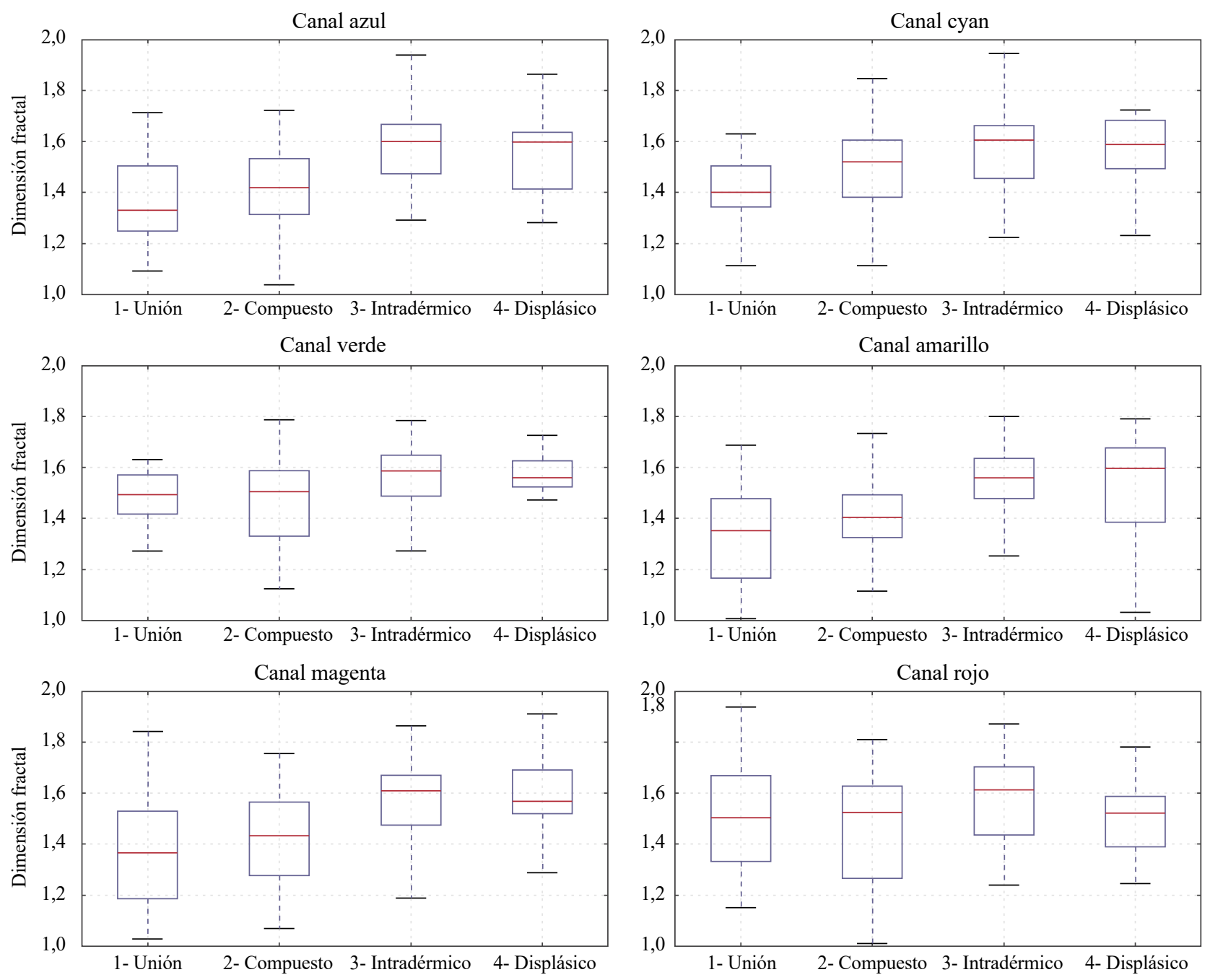

Figura 4. Gráficas de cajas de la comparación de las distribuciones en cada canal de la dimensión fractal de cada tipo de nevo melanocítico adquirido. a) Canal azul. b) Canal cyan. c) Canal verde. d) Canal amarillo. e) Canal magenta. f) Canal rojo.

correspondientes a los cuatro tipos de nevos melanocíticos adquiridos, así como la distribución de grises o histograma en los niveles de grises de cada lesión.

\section{Resultados y análisis}

Los nevos melanocíticos adquiridos se manifestaron en una imagen según la proporción de luz absorbida, lo cual depende de la presencia y la distribución de los nidos de melanina en la lesión. Para determinar si existían diferencias significativas en los NMA se hizo un análisis de varianza de los cuatro tipos: de unión, compuesto, intradérmico y displásico, en cada uno de los canales y considerando la medida de la dimensión fractal (Figura 4) y la de la entropía (Figura 5). En la Tabla 1 se resumen los resultados del ANOVA en cada canal tanto para la dimensión fractal como para la entropía. El nivel de significación fue de $\alpha=0,05$, por lo que se pudo concluir que las medidas de dimensión fractal permitieron diferenciar los grupos de NMA con un nivel de significación (p) menor que $\alpha$, excepto cuando la imagen de la lesión se obtuvo a través del canal rojo. Asimismo, la medida de la entropía también permitió diferenciar los grupos de NMA con un alto nivel de significación $(\mathrm{p}<\alpha)$, esta vez en todos los canales de observación.

En la Figura 4 se pueden observar los gráficos de cajas que representan la distribución de las medidas de la dimensión fractal por grupos de NMA en los seis canales. Resalta lo encontrado en el canal verde, en el cual los datos fueron menos dispersos que en cualquiera de los otros; sin embargo, fue en los canales azul $(p=7,87 e-8)$ y magenta $(p=7,00 e-6)$ donde se registró mayor significación en la diferencia de las medias de la dimensión fractal entre los grupos.

Las pruebas por pares se llevaron a cabo con el método de Tukey (Tabla 2). Los pares de grupos de interés fueron el de unión y displásico y el de compuesto y displásico, los cuales mostraron altos niveles de significación $(\mathrm{p}<0,05)$, excepto en los canales verde y rojo. La prueba no pudo distinguir entre lesiones intradérmicas y displásicas en ninguno de los canales. 


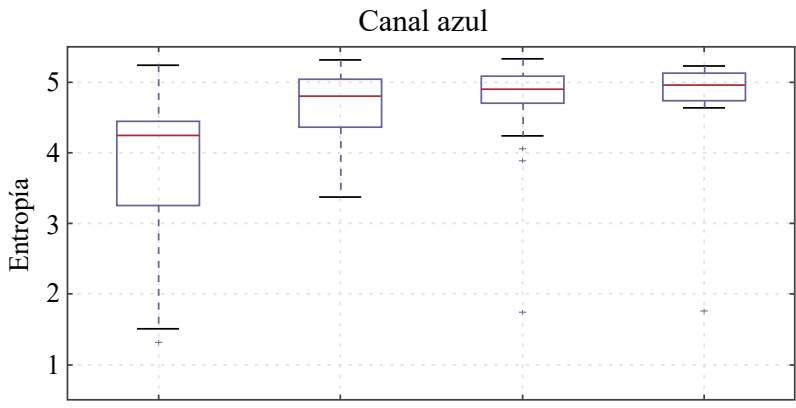

1- Unión 2-Compuesto 3-Intradérmico 4- Displásico

Canal verde

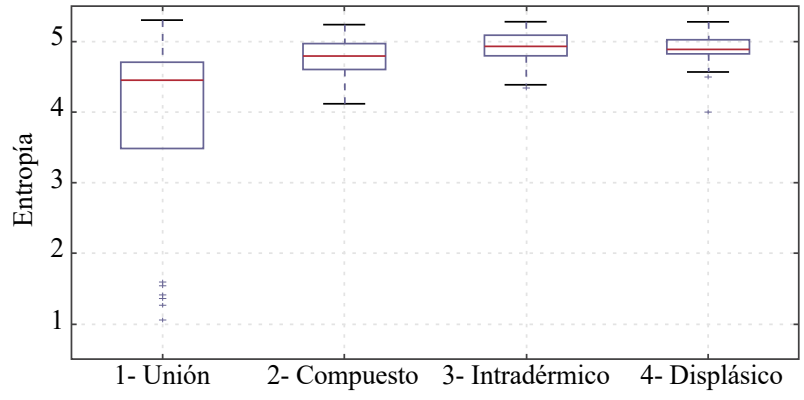

Canal magenta

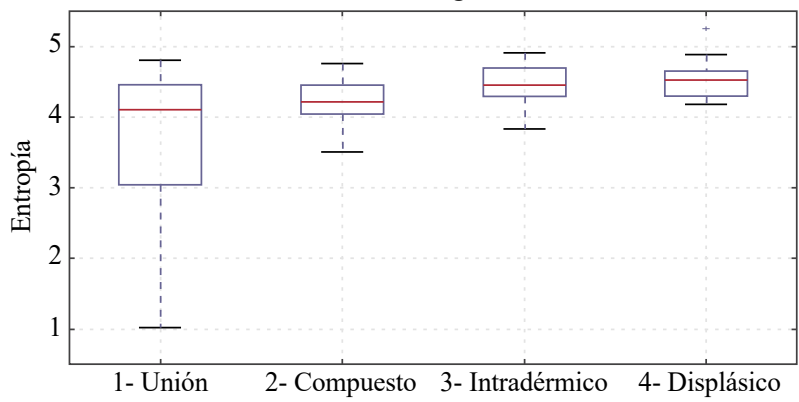

Canal cyan

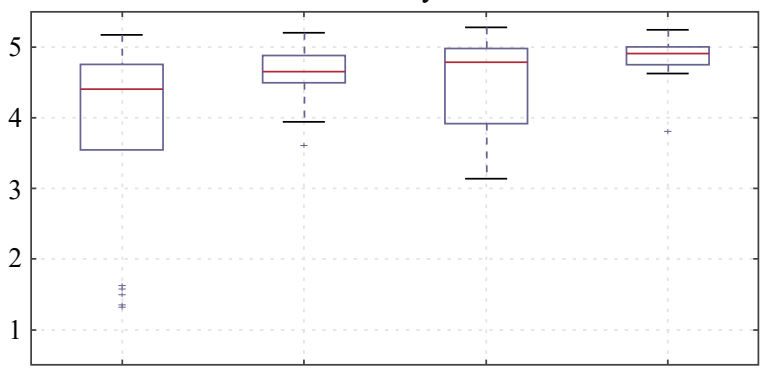

1- Unión 2-Compuesto 3-Intradérmico 4-Displásico

Canal amarillo

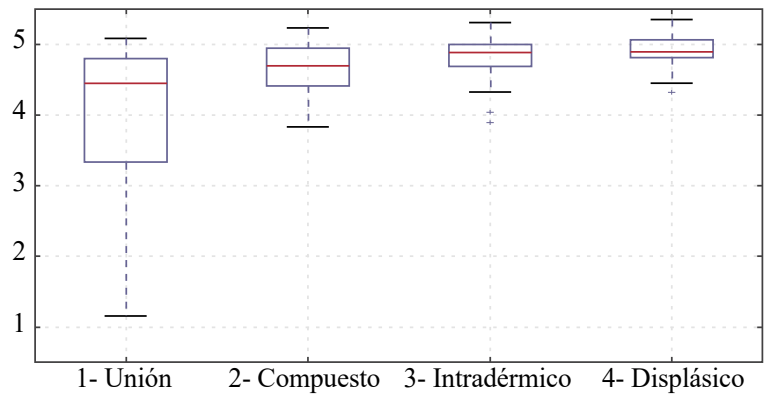

Canal rojo

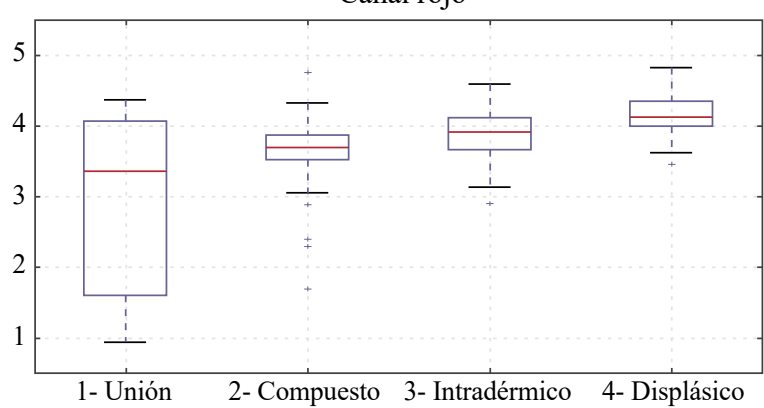

Figura 5. Gráficas de cajas de la comparación de las distribuciones en cada canal de la entropía de los tipos de nevo melanocítico adquirido. a) Canal azul. b) Canal cyan. c) Canal verde. d) Canal amarillo. e) Canal magenta. f) Canal rojo

Tabla 1. Resultados del ANOVA unifactorial con cada longitud de onda para la dimensión fractal y la entropía

\begin{tabular}{lcc}
\hline \multirow{2}{*}{ Canal } & \multicolumn{2}{c}{ Dimensión fractal } \\
\cline { 2 - 3 } & $\mathbf{p}$ & $\mathbf{F}_{(3,140)}$ \\
\hline Azul & $7,87 \mathrm{e}-08$ & 13,59 \\
Cyan & $1,28 \mathrm{e}-03$ & 5,53 \\
Verde & $5,13 \mathrm{e}-03$ & 4,45 \\
Amarillo & $1,13 \mathrm{e}-07$ & 13,10 \\
Magenta & $7,00 \mathrm{e}-06$ & 9,73 \\
Rojo & $4,85 \mathrm{e}-02$ & 2,69 \\
\hline
\end{tabular}

F: estadístico $\mathrm{F}$

Las medidas de la entropía y su dispersión pueden verse en las gráficas de cajas de la Figura 5. Resulta inconveniente usar esta medida para agrupar los lunares dada su baja significación de la homegeneidad de los datos en la prueba de
Leveine $(\mathrm{p}<0,05)$. Los resultados de la prueba T de Student en los pares de grupos de interés (unión y displásico, compuesto y displásico e intradérmico) se presentan en la Tabla 2. Se destacó la alta significación $(\mathrm{p}<0,05)$ en los análisis en los canales cyan y rojo, dado que con esta radiación se logró diferenciar los nevos de unión, compuesto e intradérmicos respecto a los displásicos.

\section{Conclusiones}

El estudio presenta una propuesta para distinguir los nevos malanocíticos adquiridos en términos del ordenamiento de los nidos de melanina vista la distribución de la energía que absorben en una imagen digital. Todos son benignos. El análisis digital de las imágenes dermatoscópicas multiespectrales permitió obtener las medidas de distribución de la energía absorbida por los Nevos melanocíticos adquiridos. Mediante la dimensión fractal se midió el orden espacial, en tanto que con la entropía se midió el orden de la distribución de grises en las imágenes. Ambas medidas se sometieron a 
Tabla 2. Resultados (p) de la comparación por pares de grupos de interés considerando la dimensión fractal y la entropía

\begin{tabular}{lcccccc}
\hline & \multicolumn{2}{c}{ Prueba de Tukey (dimensión fractal) } & \multicolumn{3}{c}{ Prueba T de Student (entropía) } \\
\cline { 2 - 7 } & UD & CD & ID & UD* & CD** & ID \\
\hline Cian & 0,002 & 0,010 & 0,900 & 0,002 & 0,023 & $0,012^{* *}$ \\
Azul & 0,002 & 0,001 & 0.900 & 0,002 & 0,503 & 0,905 \\
Verde & 0,307 & 0,195 & 0.900 & 0,002 & 0,310 & 0,011 \\
Amarillo & 0,002 & 0,015 & 0.900 & 0,001 & 0,546 \\
Magenta & 0,002 & 0,014 & 0.900 & 0,001 & $1,57 \mathrm{e}-4$ & 0,293 \\
Rojo & 0,900 & 0,730 & 0.730 & $1,0 \mathrm{e}-4$ & $2,55 \mathrm{e}-5$ \\
\hline
\end{tabular}

*Prueba de Leveine: $\mathrm{p}<0,05$ (se asumen varianzas diferentes). ** Prueba de Leveine: $\mathrm{p}>0,05$ (se asumen varianzas iguales). UD: unión-displásico, CD: compuesto-displásico, ID: intradérmico-displásico,

un ANOVA unifactorial con el cual se pudieron establecer las diferencias entre los distintos grupos de nevos melanocíticos adquiridos y el orden asociado a los tipos de NMA.

Los resultados permiten concluir que el nivel de confianza $(\mathrm{p}<0,05)$ de las comparaciones de las medidas de la dimensión fractal y la entropía permitió diferenciar los distintos grupos de NMA. Asimismo, para distinguir los nevos displásicos y los nevos melanocíticos comunes se pudo seleccionar un canal específico, por ejemplo, los canales cyan y rojo para la entropía.

Las imágenes multiespectrales son de gran relevancia, ya que con ellas puede reconocerse la forma en que la luz interactúa con un medio estratificado como la piel humana. Así, cada longitud de onda penetra de manera distinta en la piel y permite aproximarse a una tomografía de la distribución de los nidos de melanina en los nevos melanocíticos adquiridos en cada capa de la piel.

\section{Contribución de los autores}

Ariana C. Di Lorenzo: realizó la revisión bibliográfica, procesado y análisis de las imágenes, análisis de resultados y redacción del texto. Reimer A. Romero: planeación del trabajo, participó en análisis de las imágenes y de resultados, redacción de texto y revisión general. Aarón Muñoz: planeación y evaluación óptica el trabajo, participó en análisis de los resultados y revisión general. Sandra C. Vivas: asesoró el parte dermatológico del trabajo, participó en análisis de los resultados y revisión general. Irene Paredes: participó en la preparación metodológica del registro de pacientes, validación patológica de los mismos y la adquisición de imágenes.

El trabajo fue presentado en la modalidad de póster en el XV Encuentro Nacional de Óptica y VI Conferencia Andina y del Caribe en Óptica y sus aplicaciones (ENO-CANCOA), evento llevado a cabo en la Universidad Industrial de Santander sede Bucarica, en Bucaramanga, Colombia del 20 al 24 de noviembre de 2017.

\section{Conflicto de intereses}

Los autores declaran no tener conflicto de intereses.

\section{Referencias}

Air University. Space Primer (2003). 'Multispectral Imagery. Fecha de consulta: 10 de octubre de 2017. Disponible en: http://www.au.af.mil/au/awc/space/primer/index.htm

Amelard R., Glaister J., Wong A., Clausi D.A. (2014). Melanoma Decision Support Using Lighting-Corrected Intuitive Feature Models. In: Scharcanski J., Celebi M. Computer Vision Tech-niques for the Diagnosis of Skin Cancer. Jacob Scharcanski, M. Emre Celeb, Springer Berlin Heidelberg. Series in BioEngineering. Springer, Berlin, Heidelberg.

Argenziano, G. \& Soyer, P. (2001). 'Dermoscopy of pigmented skin lesions - a valuable tool for early detection. The Lancet Oncology. 2 (7): 443-449.

Brieva, J. A. \& Montes, L. (1995). EI Análisis de entropía. Un método para determinar el grado de selección en un sedimento. Aplicación en un área del Caribe colombiano". GEOCOL. 19: 145-151.

Cavalcanti P.G. \& Scharcanski J. (2014) Texture Information in Melanocytic Skin Lesion Analysis Based on Standard Camera Images. In: Scharcanski J., Celebi M. (Editors). Computer Vision Techniques for the Diagnosis of Skin Cancer. Series in BioEngineering. Springer, Berlin, Heidelberg.

Chang WY, Huang A, Yang CY, Lee CH, Chen YC, Wu TY, Chen GS. (2013). Computer-aided diagnosis of skin lesions using conventional digital photography: A reliability and feasibility study. PLoS One, 8 (11).

De Giorgi, V., Piccolo, D., Argenziano, G., Soyer, P. (2004). Interactive atlas of dermoscopy'. JAAD. 50 (5): 807-808.

Estrada G. \& William F. (2004). Geometría fractal: conceptos y procedimientos para la construcción de fractales. 1a Edición, Bogotá, D.C. Colombia, Cooperativa Editorial Magisterio.

Falconer, K. (2007) Fractal Geometry: Mathematical Foundations and Applications'. UK, Wiley.

Fodor, L., Ullmann, Y., Elman, M. (2012). Aplicaciones estéticas de la luz pulsada intensa'. Alemania, Editorial Amolca.

González, R.C.\& Woods, R.E.(2008). Digital Image Processing. New Jersey, USA, Pearson Prentice Hall.

International Agency for Research on Cancer (IARC). (2012). GLOBOCAN 2012: Estimated Cancer Incidence, Mortality and Prevalence Worldwide in 2012'. Fecha de consulta: 10 de abril de 2018. Disponible en: http://globocan.iarc.fr

Isasi A. G., Zapirain B. G., Zorrilla A. M. (2011). Melanomas non-invasive diagnosis application based on the ABCD rule and pattern recognition image processing algorithms. Comput Biol Med. 41 (9): 742-55. 
Kapsokalyvas D., Bruscino N., Alfieri D., de Giorgi V., Cannarozzo G., Cicchi R., Massi D., Pimpinelli N., Pavone F. S. (2013). Spectral morphological analysis of skin lesions with a polarization multispectral dermoscope. Optics Express. 21 (4): 4826-4840.

Kim S.,Cho D.,Kim J., Kim M., Youn S., Jang J. E., Je M., Lee D. H., Lee B.,Farkas D. L., Hwang J. Y. (2016). Smartphonebased multispectral imaging: System development and potential for mobile skin diagnosis. Biomedical Optics Express. 7 (12): 5294-5307.

Manousakia A. G., Manios A. G., Tsompanakic E. I., Tosca A. D. (2016). Use of color texture in determining the nature of melanocytic skin lesions - a qualitative and quantitative approach. Computers in Biology and Medicine. 36 (4): 419-427.

Ruela, M., Barata, C., Marques, J.S, Rozeira, J. (2015). A system for the detection of melanomas in dermoscopy images using shape and symmetry features. CMBBE: Imaging \& Visualization. 5 (2): 127-137.

McIntosh L. M., Summers R., Jackson M., Mantsch H. H., Mansfield J. R., Howlett M., Crowson A. N., Toole J.W.P. (2001). Towards Non-Invasive Screening of Skin Lesions by Near-Infrared Spectroscopy. Journal of Investigative Dermatology. 116 (1): 175-181.

Moreno-Giménez, J.C., Garnacho-Saucedo, G.M. (2012), Trastornos de la pigmentación: léntigos, nevos y melanoma. Fotoprotección. Pediatría Integral. 16 (4): 321-331.

Muñoz, A. \& Vivas, S. (2014). Implementación de sistema de captura de imágenes multiespectrales para el análisis de la piel humana. FARAUTE. 9 (1).
Paredes, I., Rabzelj B., Vivas S., Muñoz A. (2016) Microscopía de epiluminiscencia multiespectral. Fecha de consulta: 10 de octubre de 2017. Disponible en: https://www. researchgate.net/publication/284187217_microscopia_de epiluminiscencia_multiespectral

Pluim, J. P. W., Maintz, J. B. A., Viergever, M. A. (2003). MutualInformation-Based Registration of Medical Images: A Survey. IEEE Transactions on Medical Imaging. 22 (8).

Rex Cavallé, J., Ferrándiz Foraster, C. Editors. (2007). Nevos melanocíticos. Fecha de consulta: 10 de octubre de 2017. Disponible en: http://www.aeped.es/sites/default/files/ documentos/Nevos.pdf

Ruiz, M. E., Palomino, N., Contreras, W. (2010). Procesamiento digital de textura: técnicas utilizadas en aplicaciones actuales de cbir. RISI. 7 (I): 57-64.

Schmerling R.A., Loria D., Cinat G., Ramos W.E., Cardona A.F., Sánchez J.L., et al. (2011). Cutaneous melanoma in Latin America: The need for more data. Rev Panam Salud Pública. 30 (5):431-8.

Sortino-Rachou, Curado M.P., Cancela M. de C. (2011). Cutaneous melanoma in Latin America: A populationbased descriptive study. Cad Saúde Publica. 27 (3):565-72.

The Cancer Atlas website. (2008). Explore Data. Fecha de consulta: 10 abril, 2018. Disponible en: http://canceratlas. cancer.org/

Vivas T. S. \& Muñoz M. (2016). Tomografía óptica multiespectral cutánea con imagej. OPA. 49 (4):219-224.

Wolff, K., Goldsmith, L. A., Katz S. I., Gilchrest B. A., Paller A. S., Leffel D. J. (2010). Dermatología en medicina general. 7a Edición. Buenos Aires. Editorial Panamericana. 